\title{
Finding a role for cancer vaccines in pancreatic cancer: a model of resilience
}

\author{
Gentry King $^{1,2}$, Steven Green ${ }^{1}$, E. Gabriela Chiorean ${ }^{1,2}$ \\ ${ }^{1}$ Department of Medicine, Division of Medical Oncology, University of Washington School of Medicine, Seattle, WA, USA; ${ }^{2}$ Clinical Research \\ Division, Fred Hutchinson Cancer Research Center, Seattle, WA, USA \\ Correspondence to: E. Gabriela Chiorean, MD. 825 Eastlake Ave East, Seattle, WA 98109, USA. Email: gchiorea@uw.edu. \\ Comment on: Hewitt DB, Nissen N, Hatoum H, et al. A Phase 3 Randomized Clinical Trial of Chemotherapy With or Without Algenpantucel-L \\ (HyperAcute-Pancreas) Immunotherapy in Subjects with Borderline Resectable or Locally Advanced Unresectable Pancreatic Cancer. Ann Surg. \\ $2022 ; 275: 45-3$.
}

Submitted Nov 18, 2021. Accepted for publication Nov 30, 2021.

doi: 10.21037/hbsn-2021-26

View this article at: https://dx.doi.org/10.21037/hbsn-2021-26

Neoadjuvant chemotherapy with folinic acid, 5-fluorouracil, irinotecan and oxaliplatin (FOLFIRINOX) or gemcitabine plus nab-paclitaxel (Gem-nabP) for 4 to 6 months, potentially followed by chemoradiotherapy and an attempt for surgical resection for select patients, represents the multidisciplinary care standard for borderline resectable (BRPC) and locally advanced pancreatic ductal adenocarcinoma (LAPC) patients with good performance status $(1,2)$. With neoadjuvant therapy, resection rates average $65 \%$ and survival ranges from 22-29 months for BRPC, whereas LAPC patients have resection rates of $15-$ $20 \%$ and survival of 16-24 months, respectively (3-5).

Several immune-based strategies have been tested to improve outcomes in pancreatic ductal adenocarcinomas (PDA). Single-agent or combination immuno-, chemo-, and radio-immunotherapies, including anti-PD1, anti-CTLA 4 , oncolytic viruses, cancer vaccines, and $\mathrm{T}$ cells adoptive immunotherapies have been unsuccessful to date for treating advanced disease, where most of the efforts were spent (6). In Annals of Surgery, Hewitt et al recently reported results of the phase III PILLAR study with HyperAcute ${ }^{\mathrm{R}}$ Pancreas algenpantucel-L (HAPa) vaccine in combination with FOLFIRINOX or gemcitabine/nab-paclitaxel and chemoradiotherapy (CRT) for the neoadjuvant treatment of patients with BRPC and LAPC (7). HAPa is a whole cell, allogeneic vaccine composed of two human PDA cell lines genetically engineered to express the a-galactosyl $(\alpha \mathrm{Gal})$ epitope (8). The initial immune response to HAPa targets the $\alpha \mathrm{Gal}$ epitopes, but immune effector cells are expected to react against other pancreatic tumor associated antigens (TAA) due to epitope spreading. Significant work led to the clinical development of HAPa in PDA. In an initial phase II study, NLG0205 in resected PDA patients, HAPa was combined with adjuvant gemcitabine and CRT. Patients treated with HAPa 300 million cells/dose had an encouraging 1-year disease free survival (DFS) of $81 \%$, which compared favorably with the $50 \%$ rate observed in RTOG-9704 (9). Moreover, immune biomarker analyses noted higher overall survival (OS) of 36 vs. 17 months among patients with increased anti-calreticulin, antimesothelin, anti-CEA or anti- $\alpha \mathrm{Gal}$ antibodies titers $v s$. none (10). These results prompted the phase III IMPRESS study with adjuvant gemcitabine and CRT with or without $\mathrm{HAPa}$ for resected PDA. Unfortunately, OS was similar in both arms: medians 30 vs. 27 months, and 3-year OS 41\% vs. $42 \%$ with control vs. HAPa, respectively. Despite an enormous effort, with 722 patients accrued between 2010 and 2013, this study's results were never published, and no translational biomarkers analyses were performed (11). While NLG0205 and IMPRESS used gemcitabine as chemotherapy backbone, PILLAR, which accrued 303 patients with BRPC and LAPC between 2013 and 2015, tested multiagent FOLFIRINOX or Gem-nabP in combination with HAPa (7). Hewitt and colleagues must be applauded for bringing this study to press (Figure 1). Neoadjuvant therapy consisted of FOLFIRINOX for five 2-week cycles or Gem-nabP for three 4-week cycles, and if no progression, fluoropyrimidine-based CRT over $51 / 2$ 


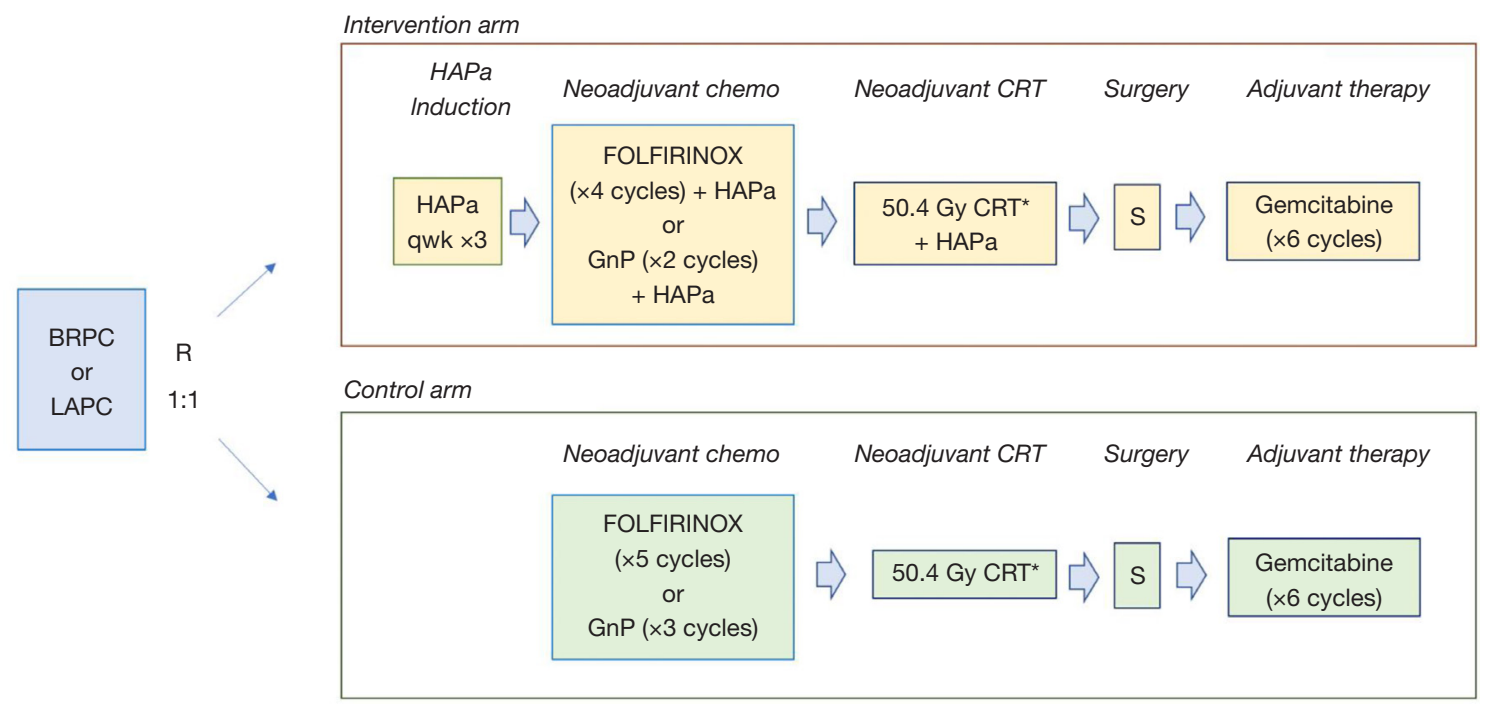

Figure 1 PILLAR study design. *, either 5-FU or capecitabine-based CRT; 1 cycle FOLFIRINOX =2 weeks; 1 cycle GnP =4 weeks. BRPC, borderline resectable pancreas cancer; LAPC, locally advanced unresectable pancreas cancer; FOLFIRINOX, folinic acid, 5-fluorouracil, irinotecan and oxaliplatin; GnP, gemcitabine and nab-paclitaxel; HAPa, HyperAcute ${ }^{\mathrm{R}}$-Pancreas algenpantucel-L; CRT, chemoradiotherapy; S, surgery.

weeks in the control arm. In the experimental arm, HAPa (300 million cells/dose) was administered weekly $\times 3$ as induction, followed by every 2 weeks in combination with FOLFIRINOX (four cycles) or Gem-nabP (two cycles), and with CRT. Patients deemed surgically resectable were offered surgical resection, followed by six 4-week cycles of adjuvant gemcitabine. Once again, no survival benefit was observed from the addition of HAPa. While the population was heterogenous, the majority of patients had LAPC (82\%), and received neoadjuvant FOLFIRINOX and Gem-nabP in 2:1 ratio, albeit for a shorter duration (2 months) than the 4-6 months currently recommended by NCCN, and ASCO guidelines $(1,2)$. The primary endpoint of OS was similar in both arms: 14.9 vs. 14.3 months $(\mathrm{HR}=1.02 ; \mathrm{P}=0.98)$ with control vs. HAPa. Likewise, progression-free survival (PFS) of $13.4 v s .12 .4$ months $(\mathrm{HR}=1.13 ; \mathrm{P}=0.59)$, and resection rates of $26 \%$ vs. $23 \%$ were similar with control vs. HAPa $(\mathrm{P}=0.52)$. PFS and OS rates were comparable for BRPC vs. LAPC, but this may have been due to low numbers of BRPC patients. Survival rates in PILLAR were lower than in other contemporary analyses (3-5), likely due to suboptimal neoadjuvant chemotherapy exposure for a patient population with mostly locally advanced, unresectable disease. Another limitation of this study is that, despite existing knowledge of the importance of eliciting an antibody or cellular antitumor immune response, no translational immune biomarker analyses were performed. In all, randomized studies showed that when added to any chemotherapy or CRT regimen, HAPa failed to improve efficacy for patients with localized pancreatic cancers. Along with IMPRESS, and the multitude of other negative vaccine studies, results from PILLAR underscore the complexities of translating cancer vaccines into successful treatments for PDA.

While it is well known that a robust anti-tumor immune response resulting in effective cytotoxicity requires tumor-associated antigen (TAA)/neoantigen recognition, immune cells activation and access into the tumor microenvironment (TME), the two major hurdles affecting vaccines efficacy have been the immunosuppressive TME and the high variability of TAAs. The former is particularly relevant in PDA as the desmoplastic stroma has been shown to not only impede the penetration of cytotoxic chemotherapy, but restrict the infiltration and activity of CD8+ T-cells that directly mediate anti-tumor activity (12). Unfortunately, data describing the stroma and immune TME from resected specimens in the $24 \%$ of patients who underwent surgery in PILLAR were not reported. With regards to TAAs, negative results of multiple randomized PDA vaccine trials highlight the difficulty of identifying specific antigens that break immune tolerance and consistently elicit quality adaptive immune responses. 
Several TAAs and neoantigens have been undergoing clinical investigation, including KRAS mutations, survivin, MUC1 and mesothelin, to name a few $(6,13)$. HAPa and pancreas GVAX employed a more inclusive TAA strategy by using a whole-cell strategy from allogeneic PDA cell lines. However, PDA antigens are, in essence, self-antigens and unlike foreign antigens like viruses, may escape immune cells recognition due to central and peripheral tolerance (13). Adjuvants and stimulators, such as the $\alpha \mathrm{Gal}$ hyperacute rejection strategy, were utilized to break the immune tolerance by stimulating low affinity and rare TAA-reactive T-cells to amplify and expand. However, these negative clinical trials suggest that the current portfolio of TAAs and stimulators may not be strong enough to overcome tolerance. Lastly, another factor that must be taken into consideration is how chemotherapy and radiotherapy regimens impact the generation and maintenance of a PDA specific immune response. PILLAR was a multi-arm study that employed the two most active chemotherapy regimens, FOLFIRINOX and GemnabP. Studies have shown that these regimens modulate the immune TME to decrease immunosuppressive T-regulatory (Tregs) and myeloid derived suppressor cells (MDSCs), resulting in increased anti-tumor CD8+ T-cell infiltration (12). Immune biomarker studies in resected tumor samples in SWOG S1505, a randomized study of perioperative FOLFIRINOX or Gem-nabP for resectable PDA, suggested a trend for increased CD68+ myeloid cells in tumors treated with FOLFIRINOX compared to GemnabP (14). As such, the optimal sequencing of standard therapies with vaccines and immunotherapies, in general remains to be defined.

In conclusion, pancreatic cancer has demonstrated profound resilience against immunotherapeutic approaches. Nevertheless, with our current, deeper understanding on the complexities of immune regulation, continuing efforts will span beyond its multi-disciplinary care to include complementary methods to enhance immune activation while counteracting immunosuppression. To this end, clinical trials with personalized, neoantigen pancreatic cancer vaccines in combination with immune checkpoint inhibitors are underway (e.g., NCT04161755, NCT04117087, NCT03956056), awaiting long-overdue success.

\section{Acknowledgments}

Funding: None.

\section{Footnote}

Provenance and Peer Review: This article was commissioned by the editorial office, Hepatobiliary Surgery and Nutrition. The article did not undergo external peer review.

Conflicts of Interest: All authors have completed the ICMJE uniform disclosure form (available at https://hbsn. amegroups.com/article/view/10.21037/hbsn-2021-26/coif). The authors have no conflicts of interest to declare.

Ethical Statement: The authors are accountable for all aspects of the work in ensuring that questions related to the accuracy or integrity of any part of the work are appropriately investigated and resolved.

Open Access Statement: This is an Open Access article distributed in accordance with the Creative Commons Attribution-NonCommercial-NoDerivs 4.0 International License (CC BY-NC-ND 4.0), which permits the noncommercial replication and distribution of the article with the strict proviso that no changes or edits are made and the original work is properly cited (including links to both the formal publication through the relevant DOI and the license). See: https://creativecommons.org/licenses/by-nc-nd/4.0/.

\section{References}

1. Balaban EP, Mangu PB, Khorana AA, et al. Locally advanced, unresectable pancreatic cancer: American Society of Clinical Oncology clinical practice guideline. J Clin Oncol 2016;34:2654-68.

2. Tempero MA, Malafa MP, Al-Hawary M, et al. Pancreatic adenocarcinoma, version 2.2021, NCCN clinical practice guidelines in oncology. J Natl Compr Canc Netw 2021;19:439-57.

3. Janssen QP, Buettner S, Suker M, et al. Neoadjuvant FOLFIRINOX in patients with borderline resectable pancreatic cancer: a systematic review and patient-level meta-analysis. J Natl Cancer Inst 2019;111:782-94.

4. Suker M, Beumer BR, Sadot E, et al. FOLFIRINOX for locally advanced pancreatic cancer: a systematic review and patient-level meta-analysis. Lancet Oncol 2016;17:801-10.

5. Williet N, Petrillo A, Roth G, et al. Gemcitabine/nabPaclitaxel versus FOLFIRINOX in locally advanced pancreatic cancer: a European multicenter study. Cancers (Basel) 2021;13:2797.

6. Timmer FEF, Geboers B, Nieuwenhuizen S, et al. 
Pancreatic cancer and immunotherapy: a clinical overview. Cancers (Basel) 2021;13:4138.

7. Hewitt DB, Nissen N, Hatoum H, et al. A Phase 3 Randomized Clinical Trial of Chemotherapy With or Without Algenpantucel-L (HyperAcute-Pancreas) Immunotherapy in Subjects with Borderline Resectable or Locally Advanced Unresectable Pancreatic Cancer. Ann Surg. 2022;275:45-3.

8. Coveler AL, Rossi GR, Vahanian NN, Link C, Chiorean EG. Algenpantucel-L immunotherapy in pancreatic adenocarcinoma. Immunotherapy 2016;8:117-25.

9. Hardacre JM, Mulcahy M, Small W, et al. Addition of algenpantucel-L immunotherapy to standard adjuvant therapy for pancreatic cancer: a phase 2 study. J Gastrointest Surg 2013; 17:94-100.

10. Rossi GR, Rocha Lima CMS, Hardacre JM, et al. Correlation of anti-calreticulin antibody titers with improved overall survival in a phase 2 clinical trial of algenpantucel-L immunotherapy for patients with resected pancreatic cancer. J Clin Oncol 2014;32:abstr 3029.

11. Ames ING. NewLink Genetics announces results from phase 3 IMPRESS trial of algenpantucel-L for patients with resected pancreatic cancer. May 9. 2016. Available online: https://www.globenewswire.com/newsrelease/2016/05/09/837878/0/en/NewLink-GeneticsAnnounces-Results-from-Phase-3-IMPRESS-Trial-ofAlgenpantucel-L-for-Patients-with-Resected-PancreaticCancer.html

12. Reyes CM, Teller S, Muckenhuber A, et al. Neoadjuvant therapy remodels the pancreatic cancer microenvironment via depletion of protumorigenic immune cells. Clin Cancer Res 2020;26:220-31.

13. Hollingsworth RE, Jansen K. Turning the corner on therapeutic cancer vaccines. NPJ Vaccines 2019;4:7.

14. Sohal D, Duong M, Chang R, et al. Immunologic predictors of therapeutic response to neoadjuvant chemotherapy for pancreatic ductal adenocarcinoma in SWOG S1505. J Clin Oncol 2021;39:abstr 419.
Cite this article as: King G, Green S, Chiorean EG. Finding a role for cancer vaccines in pancreatic cancer: a model of resilience. HepatoBiliary Surg Nutr 2022;11(1):115-118. doi: 10.21037/hbsn-2021-26 